\title{
Health Monitoring System for Mine Areas
}

\author{
Nagolu Sainadh, P. Dass
}

ABSTRACT- For underground coal mines environment should be kept monitered for the safety of people working underneath, the existing safety systems are not effective and expensive as well. The present health monitoring systems which are being used inside the coal mines need to connect through wires all the way which makes the system more expensive. Therefore wireless sensor network with various sensors to monitor all the important parameters in the mine are proposed and analyzed. Using gas sensor and temperature sensor we can derive the gas and temperature level of that place in the mine. Using heart rate sensor the pulse of the miner will be monitored continuously. Here NRF device is used to analyze the data and intimate the control unit.

\section{INTRODUCTION}

The proposed health monitoring system consists of ArduinoUno and ArduinoMega in which mega is used as miner module whereas Uno is used as control module. All the sensor networks are connected to the ArduinoMega which is preprogrammed with necessary commands to analyze the harmful parameters within the mine and as well as the body condition of the miner. The sensors like temperature sensor and heart rate sensors will work continuously to monitor the temperature and heart rate of the miner and displays the data both on the miner unit and control unit. If abnormal temperature or heart rate is detected the system alerts both the control unit and the miner to take the necessary steps to avoid any hazardous situations. NRF transceiver is used at both the ends for effective transmission of data between miner and control unit. The NRF transceivers are aligned with same address to receive and transfer data. The important factor for using NRF transceiver is device to device communication. In this communication system for longer distances more number of NRF devices can be installed where each device will communicate to the next nearest device and the next one will communicate to the next nearest device towards the destination. Therefore this system is more effective and efficient even for longer distances.

\section{WORKING PRINCIPLE}

Here we proposed a propelled specialized gadget for the coal laborers. In this venture we put a few sensors and controllers to screen nature. Pulse and temperature sensor give the wellbeing status of the laborer. Here we utilized a few switches. Utilizing this we send the crisis and some critical predefined messages. The temperature sensors are

Revised Manuscript Received on August 14, 2019.

Nagolu Sainadh, UG Scholar, ECE Department, Saveetha School of Engineering (SIMATS), Tamil Nadu, India (Email: sainadh.m123@gmail.com)

P. Dass, Assistant Professor, UG Scholar, ECE Department, Saveetha School of Engineering (SIMATS), Tamil Nadu, India (Email: dasseie@gmail.com) assigned for both miner's body temperature and underground temperature will be monitored continuously. The water level sensor will detect any moisture inside the mine and alerts the control unit. This information will send to the control unit with the help of NRF.

\section{BLOCKDIAGRAM}

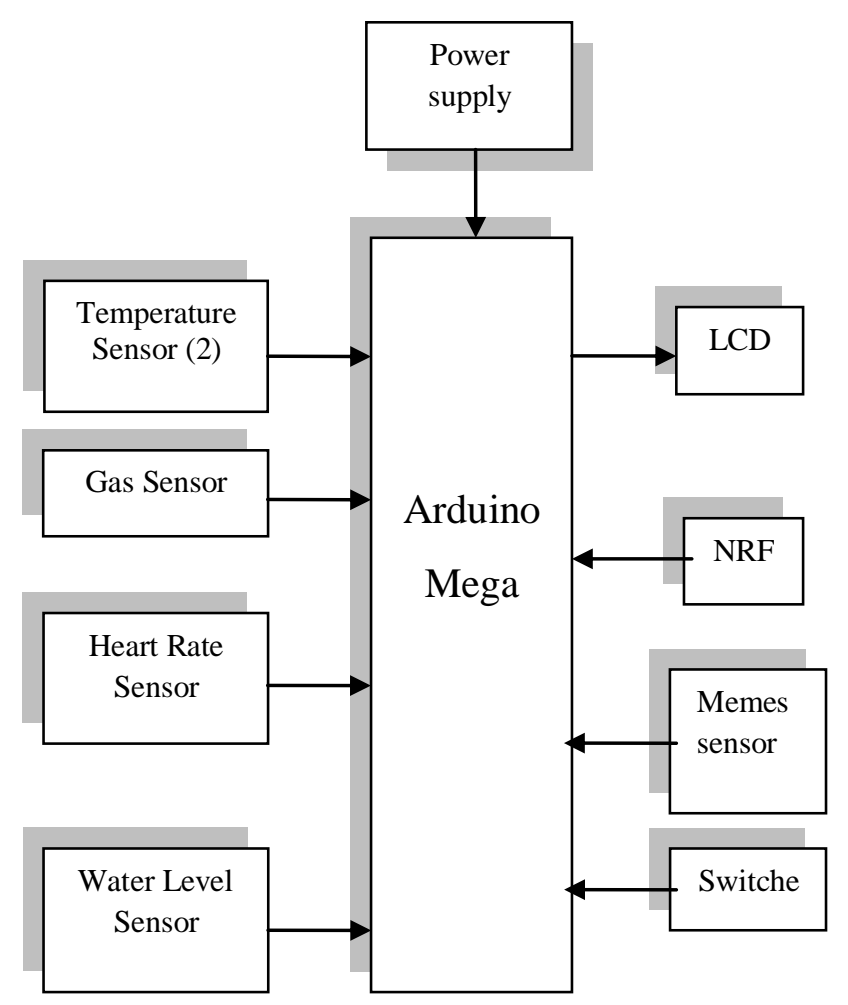

Fig.1: Miner module

CONTROL UNIT

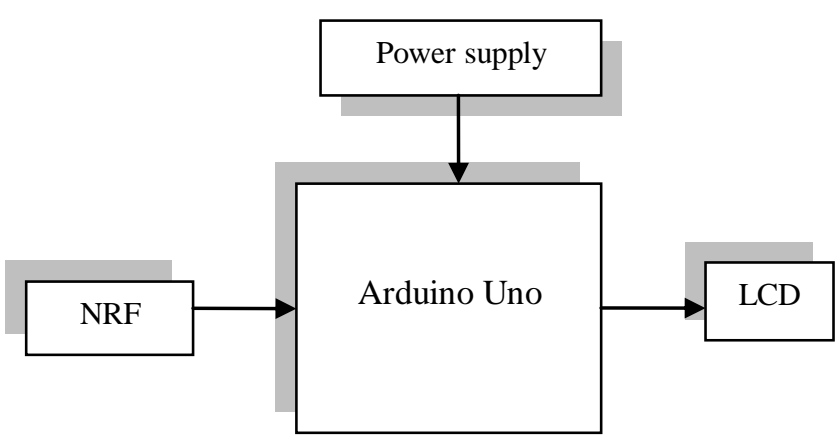

Fig.2: Control module

Published By: Blue Eyes Intelligence Engineering \& Sciences Publication

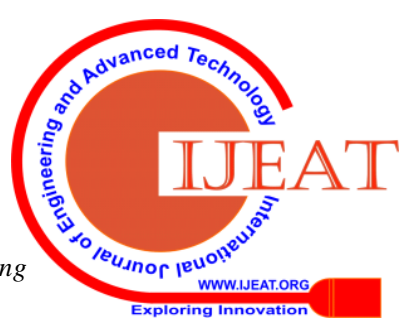


HEALTH MONITORING SYSTEM FOR MINE AREAS

\section{EXISTING AND PROPOSED SYSTEM}

\section{Existing System}

$>$ In mine, the wired communication technique is used for monitoring.

$>$ There is no wireless technology is available for monitoring.

Drawbacks

$>\quad$ There is no wireless system for data transfer.

It is not possible to monitor there sources.

$>\quad$ High cost and more time consumption

\section{Proposed System}

The advanced health management system consists of NRF based accessing technology.

$>$ The NRF technology is used to identify the particular person's data base.

We find temperature and Heart rate level of the mine workers.

$>\quad$ Here we used some Emergency switched.

Advantages:

$>\quad$ The fast data transfer.

$>$ Wireless.

$>$ Efficiency.

$>\quad$ Low cost.

\section{HARDWARE REQUIREMENTS \& RESULTS}

$\begin{array}{ll}> & \text { Arduino Mega } \\ > & \text { Arduino Uno } \\ > & \mathrm{NRF}(2) \\ > & \mathrm{LCD}(2) \\ > & \text { Switches }(2) \\ > & \text { Gas Sensor } \\ >\quad \text { Heart rate sensor } \\ >\quad \text { Water level sensor } \\ \quad \text { Temperature sensor(2) }\end{array}$

\section{Arduino Mega}

The Mega 2560 is microcontroller board dependent on the ATmega2560. It has 54 computerized information/yield pins (of which 15 can be utilized as PWM yields), 16 simple sources of info, 4 UARTs (equipment sequential ports), a 16 $\mathrm{MHz}$ gem oscillator, a USB association, a power jack, an ICSP header, and a reset catch. It contains everything expected to help the microcontroller; just interface it to a PC with a USB link or power it with an AC-to-DC connector or battery to begin. The Mega 2560 board is perfect with most shields intended for the Uno and the previous sheets Duemilanove or Diecimila.

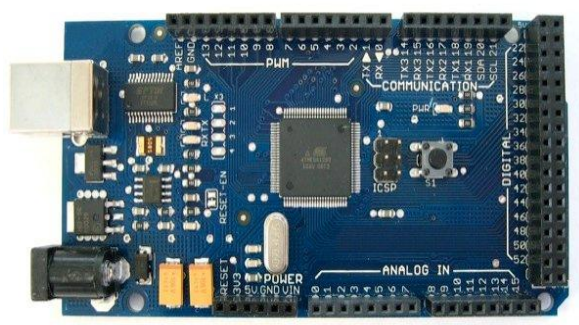

Fig.3: Arduino Mega

\section{Programming}

The Mega 2560 board can be customized with the Arduino Software (IDE). For subtleties, see the reference and instructional exercises. The ATmega2560 on the Mega 2560 comes prearranged with a boot loader that enables you to transfer new code to it without the utilization of an outer equipment developer. It imparts utilizing the first STK500 convention (reference, C header documents). You can likewise sidestep the bootloader and program the microcontroller through the ICSP (In-Circuit Serial Programming) header utilizing Arduino ISP or comparable; see these guidelines for subtleties. The ATmega16U2 (or $8 \mathrm{U} 2$ in the rev1 and rev2 sheets) firmware source code is accessible in the Arduino store. The ATmega16U2/8U2 is stacked with a DFU bootloader, which can be enacted by: a. On Rev1 sheets: associating the patch jumper on the back of the board (close to the guide of Italy) and afterward resetting the $8 \mathrm{U} 2$.

b. On Rev2 or later sheets: there is a resistor that pulling the 8U2/16U2 HWB line to ground, making it simpler to put into DFU mode. You would then be able to utilize Atmel's FLIP programming (Windows) or the DFU developer (Mac OS $X$ and Linux) to stack another firmware. Or on the other hand you can utilize the ISP header with an outside developer (overwriting the DFU bootloader). See this client contributed instructional exercise for more data.

\section{Arduino Uno}

Arduino/Genuino Uno is a microcontroller board dependent on the ATmega328P. It has 14 computerized information/yield pins (of which 6 can be utilized as PWM yields), 6 simple data sources, a $16 \mathrm{MHz}$ quartz precious stone, a USB association, a power jack, an ICSP header and a reset catch. It contains everything expected to help the microcontroller; just associate it to a PC with a USB link or power it with an AC-to-DC connector or battery to begin. You can tinker with your UNO without stressing a lot over accomplishing something incorrectly, most dire outcome imaginable you can swap the chip for a couple of dollars and begin once again once more.

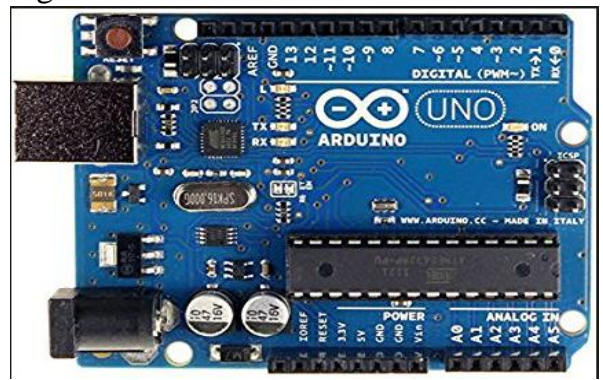

Fig.4: Arduino UNO

"Uno" signifies one in Italian and was picked to check the arrival of Arduino Software (IDE) 1.0. The Uno board and form 1.0 of Arduino Software (IDE) were the reference renditions of Arduino, presently developed to more up to date discharges. The Uno board is the first in a progression 
of USB Arduino sheets, and the reference display for the Arduino stage; for a broad rundown of current, past or obsolete sheets see the Arduino list of sheets.

\section{Programming}

The Arduino/Genuino Uno can be customized with the (Arduino Software (IDE)). Select Arduino/Genuino Uno from the Tools $>$ Board menu (as per the microcontroller on your board). For subtleties, see the reference and instructional exercises. The ATmega328 on the Arduino/Genuino Uno comes prearranged with a bootloader that enables you to transfer new code to it without the utilization of an outer equipment software engineer. It imparts utilizing the first STK500 convention (reference, C header records). You can likewise sidestep the bootloader and program the microcontroller through the ICSP (InCircuit Serial Programming) header utilizing Arduino ISP or comparative; see these guidelines for subtleties. The ATmega16U2 (or 8U2 in the rev1 and rev2 sheets) firmware source code is accessible in the Arduino archive. The ATmega16U2/8U2 is stacked with a DFU bootloader, which can be actuated by:

On Rev1 sheets: associating the bind jumper on the back of the board (close to the guide of Italy) and after that resetting the $8 \mathrm{U} 2$.

On Rev2 or later sheets: there is a resistor that pulling the $8 \mathrm{U} 2 / 16 \mathrm{U} 2 \mathrm{HWB}$ line to ground, making it simpler to put into DFU mode.

You would then be able to utilize Atmel's FLIP programming (Windows) or the DFU software engineer (Mac OS X and Linux) to stack another firmware. Or on the other hand you can utilize the ISP header with an outside software engineer (overwriting the DFU bootloader). See this client contributed instructional exercise for more data.

\section{3. $N R F$}

The nRF24L01 is a profoundly coordinated, ultra-low power (ULP) $2 \mathrm{Mbps}$ RF handset IC for the $2.4 \mathrm{GHz}$ ISM (Industrial, Scientific and Medical) band. With pinnacle $\mathrm{RX} / \mathrm{TX}$ flows lower than $14 \mathrm{~mA}$, a sub $\mu \mathrm{A}$ shut down mode, propelled control the board, and a 1.9 to $3.6 \mathrm{~V}$ supply extend, the nRF24L01 gives a genuine ULP arrangement empowering a very long time to long stretches of battery lifetime when running on coin cells or AA/AAA batteries. The Enhanced ShockBurst ${ }^{\mathrm{TM}}$ equipment convention quickening agent moreover offloads time basic convention capacities from the application microcontroller empowering the execution of cutting edge and strong remote network with minimal effort outsider microcontrollers. The nRF24L01 facilitates an all out $2.4 \mathrm{GHz}$ RF handset, RF synthesizer, and baseband method of reasoning including the Enhanced ShockBurst ${ }^{\mathrm{TM}}$ hardware tradition stimulating operator supporting a fast SPI interface for the application controller. No outer circle channel, resonators, or VCO varactor diodes are required, just a minimal effort $\pm 60 \mathrm{ppm}$ precious stone, coordinating hardware, and recieving wire. The Nordic nRF24L01 is accessible in a minimal 20-stick 4 $\mathrm{x} 4 \mathrm{~mm}$ QFN bundle.

\section{Features}

1. Low cost single-chip 2.4ghz GFSK RF handset IC.
2. Worldwide permit free $2.4 \mathrm{ghz}$ ISM band activity $2 \mathrm{mbps}$ on-air information rate.

3. Enhanced shockburst ${ }^{\mathrm{TM}}$ equipment convention quickening agent.

4. Ultra low power utilization - months to long stretches of battery lifetime.

5. On-air good with all Nordic nRF24l series in 1 and 2 mbps mode.

6. On-air good with Nordic nRF24e and nRF240 series in $1 \mathrm{mbps}$ mode.

\section{LCD (Liquid Crystal Display)}

LCD screen is an electronic presentation module and locate a wide scope of utilizations. A 16x2 LCD show is exceptionally fundamental module and is in all respects normally utilized in different gadgets and circuits. These modules are favored more than seven sections and other multi portion LEDs. The reasons being: LCDs are efficient; effectively programmable; have no confinement of showing uncommon and even custom characters (not at all like in seven fragments), livelinesss, etc. A 16x2 LCD implies it can show 16 characters for every line and there are 2 such lines. In this LCD each character is shown in $5 \times 7$ pixel framework. This LCD has two registers, to be specific, command and data. The order register stores the direction guidelines given to the LCD. An order is a guidance given to LCD to complete a predefined task like introducing it, clearing its screen, setting the cursor position, controlling showcase and so on. The information register stores the information to be shown on the LCD. The information is the ASCII estimation of the character to be shown on the LCD. Snap to study inner structure of a LCD.

\section{Pin Diagram:}

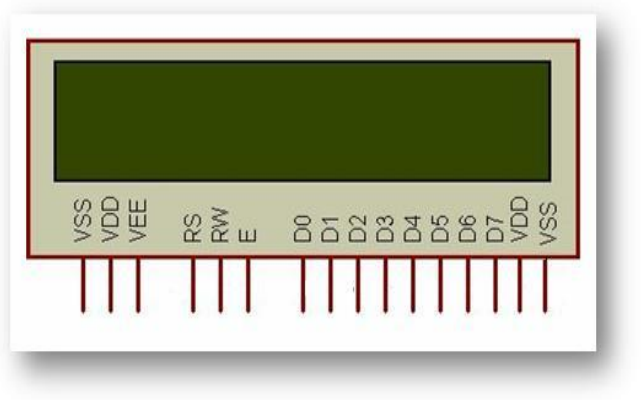

Fig.5: LCD PINS

\section{Gas Sensor:}
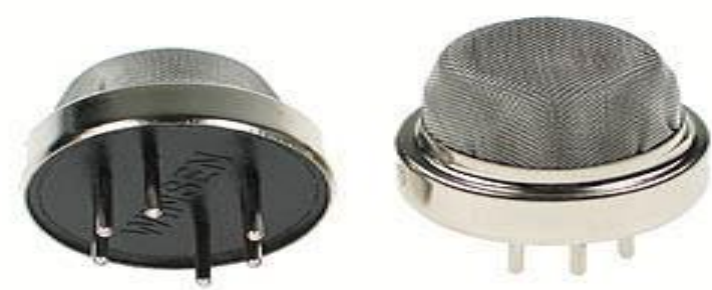

Fig.6: Gas Sensor

Published By: 


\section{Construction}

The sensors contain two in contact with an electrolyte. The cathodes are regularly manufactured by fixing a high surface zone valuable metal on to the permeable hydrophobic layer. The working cathode contacts both the electrolyte and the surrounding air to be checked generally by means of a permeable film. The electrolyte most normally utilized is a mineral corrosive the anodes and lodging are more often than not in a plastic lodging which contains a gas passage opening for the gas and electrical contacts.

\section{Hypothesis Of Operation}

The gas diffuses into the sensor, through the back of the permeable layer to the working terminal where it is oxidized or decreased. This electrochemical response results in an electric flow that goes through the outer circuit. Notwithstanding estimating, intensifying and performing other flag preparing capacities, the outer circuit keeps up the voltage over the sensor between the working and counter cathodes for a two terminal sensor or between the working and reference anodes for a three anode cell. At the counter terminal an equivalent and inverse response happens, with the end goal that in the event that the working anode is an oxidation, at that point the counter cathode is a decrease.

\section{Heart Rate Sensor}

Heart beat is detected by utilizing a high force type LED and LDR. The finger is set between the LED and LDR. As sensors photograph diode or a photograph transistor can be utilized. The skin might be lit up with obvious (red) utilizing transmitted or reflected light for identification. The exceptionally little changes in reflectivity or in transmittance brought about by the shifting blood substance of human tissue are practically undetectable. Different commotion sources may deliver unsettling influence signals with amplitudes equivalent or considerably higher than the plentifulness of the beat flag. Legitimate heartbeat estimation in this manner requires broad preprocessing of the crude flag. The new flag handling approach introduced here joins simple and advanced flag preparing such that the two sections can be kept basic however in mix are powerful in stifling aggravation signals. The setup portrayed here utilizations a red LED for transmitted light enlightenment and a LDR as finder.

\section{Water Level Sensor}

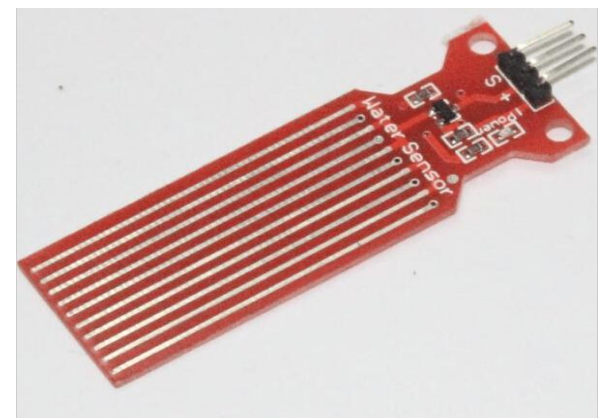

Fig.7: Water sensor

Proceeding with sensors that can be utilized in aquaponics, hydroponics frameworks or programmed open air plant care, we will disclose how to utilize a water level sensor to control the measure of water we have in a tank. A similar sensor can likewise be utilized to distinguish in the event that it is sprinkling.

\section{Temperature Sensor (LM35)}

The LM35 is an incorporated circuit sensor that can be utilized to gauge temperature with an electrical yield corresponding to the temperature (in degrees celsius). The LM35 - An Integrated Circuit Temperature Sensor. It can gauge temperature more precisely than an utilizing a thermistor. The sensor hardware is fixed and not expose to oxidation, and so on. The LM35 produces a higher yield voltage than thermocouples and may not necessitate that the yield voltage be enhanced.

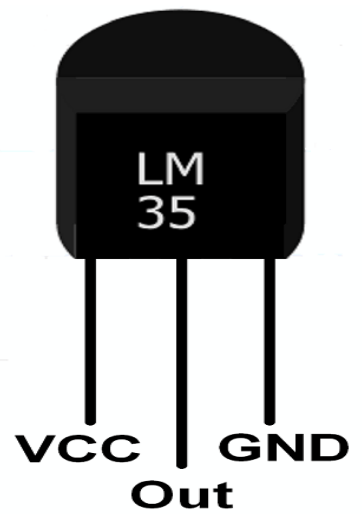

Fig.8: Temperature sensor

\section{SOFTWARE REQUIREMENTS}

$>$ Embedded C

$>$ Arduino IDE

\section{Embedded $C$ :}

Embedded C is a set of language extensions for the $\mathrm{C}$ Programming language by the $\mathrm{C}$ Standards advisory group to address shared characteristic issues that exist between $\mathrm{C}$ augmentations for various installed frameworks. Truly, installed $\mathrm{C}$ programming requires nonstandard augmentations to the $\mathrm{C}$ language so as to help intriguing highlights, for example, fixed-point number juggling, numerous unmistakable memory banks, and essential I/O activities. Inserted $\mathrm{C}$ utilizes a large portion of the linguistic structure and semantics of standard C, e.g., fundamental() work, variable definition, datatype affirmation, restrictive proclamations (if, switch case), circles (while, for), capacities, exhibits and strings, structures and association, bit tasks, macros, and so forth.

\section{Arduino IDE:}

The Arduino/Genuino Uno can be modified with the (Arduino Software (IDE)). Select "Arduino/Genuino Uno from the Tools > Board menu (as per the microcontroller on your board). For subtleties, see the reference and instructional exercises. The ATmega328 on the Arduino/Genuino Uno comes prearranged with a bootloader that enables you to transfer new code to it without the

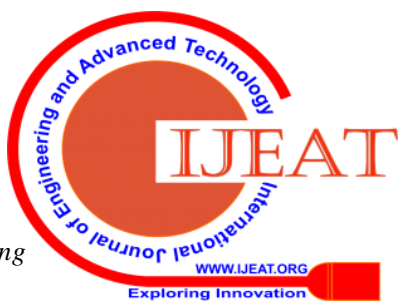


utilization of an outside equipment developer. It conveys utilizing the first STK500 convention (reference, C header documents).

\section{RESULTS}

The various results of the sensor networks are as follows:

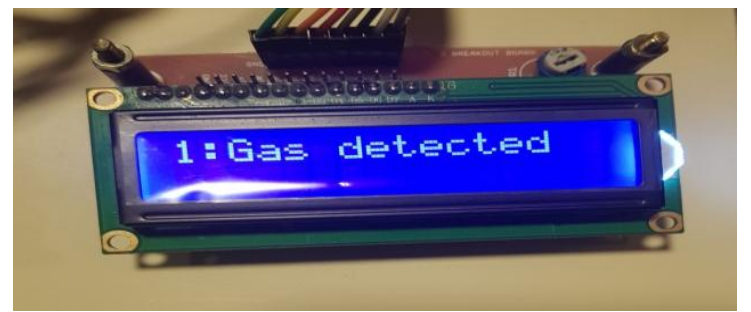

Fig.9: LCD showing gas detected

In Fig.9 the MQ4 gas sensor is assigned with a fixed amount of threshold gas level, if the gas sensor detects a gas above the level then it is displayed on the control unit LCD.

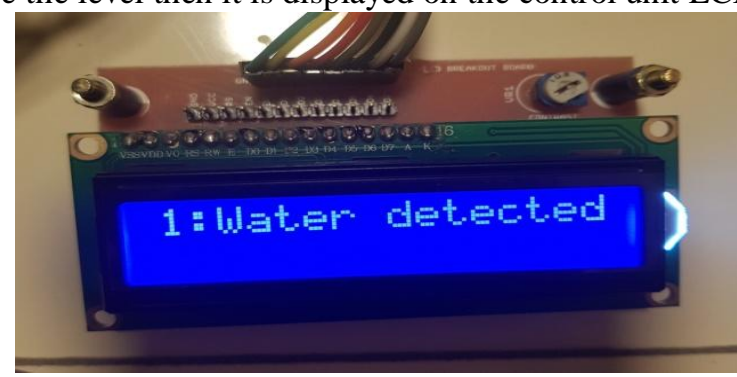

Fig.10: LCD showing water detected

The Fig.10 shows the message water detected if any moisture content detected in the mine area. The sensor alerts the control unit and displays a message.

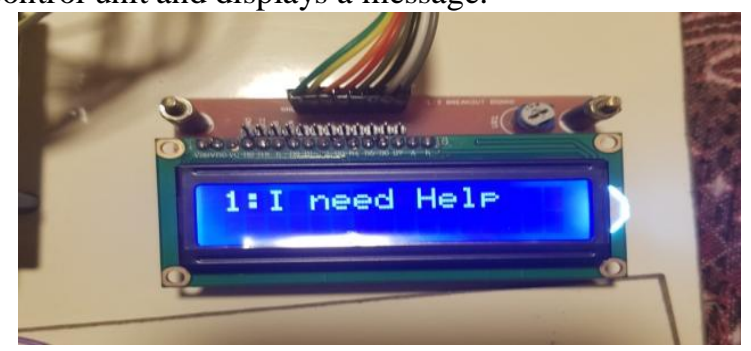

Fig.11: LCD showing emergency message

When emergency button is pressed by the miner the control unit automatically displays "I need help" on the display of control unit as shown in the Fig.11.

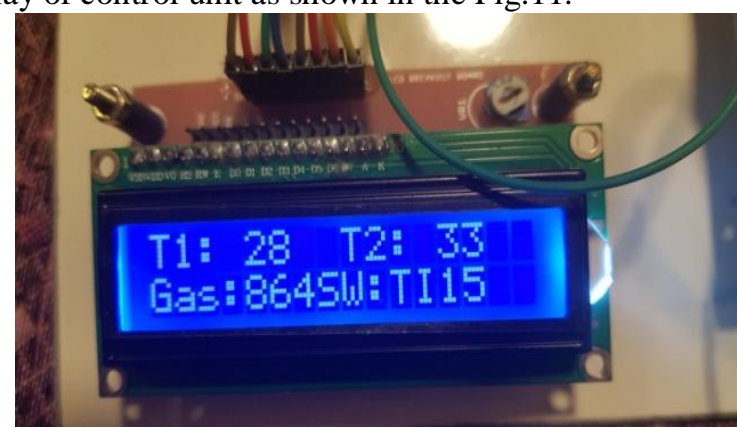

Fig.12: LCD showing temperatures

Fig. 12 shows the temperatures of mine and miner and gas levels in the mine. The LM35 sensors will keep on detecting the temperatures and displays continuously on the LCD.

\section{CONCLUSION}

The examination on continuous observing of harmful gases and different parameters present in underground mine has investigated utilizing remote sensor arrange. An ongoing checking framework is created to give more clear and more point to point viewpoint of the underground mine. This framework is showing the parameters on the LCD at the underground segment where sensor unit is introduced just as on the checking unit; it will be useful to all diggers present inside the mine to spare their life before any loss happens. Alert triggers when sensor esteems crosses the limit level. This framework additionally stores every one of the information in the $\mathrm{PC}$ for future examination.

\section{FUTURE SCOPE}

1. Utilizing extra sensors all conceivable wellbeing issues could be observed, for example, gases, dust, vibrations, fire and so forth

2. The other critical information can be conveyed through this framework making it possible where wired correspondence is an obstacle.

3. The control can be administered from the surface itself as the framework gives simple access.

\section{REFERENCES}

1. Asadi, Q. Wang, V. Mancuso, "A survey on device-todevice communication in cellular networks," Communications Surveys \& Tutorials IEEE, vol. 16, no. 4, pp. 1801-1819. doi: 10.1109/COMST.2014.2319555.

2. Virdis, C. Vallati, G. Nardini, G. Tanganelli, G. Stea and E. Mingozzi, "D2D Communications for Large-Scale Fog Platforms: Enabling Direct M2M Interactions," IEEE Vehicular Technology Magazine, vol. 13, no. 2, pp 24-33, June 2018. doi: 10.1109/MVT.2017.2775560

3. O. Bello and S. Zeadally, "Intelligent Device-to-Device Communication in the Internet of Things," IEEE Systems Journal, vol. 10, no. 3, pp. 1172-1182, Sept. 2016. doi: 10.1109/JSYST.2014.2298837.

4. J. Kang, R. Yu, X. Huang and Y. Zhang, "PrivacyPreserved Pseudonym Scheme for Fog Computing Supported Internet of Vehicles," in IEEE Transactions on Intelligent Transportation Systems. doi 10.1109/TITS.2017.2764095

5. R. Sutton and A. Barto, Reinforcement Learning: An Introduction, Adaptive Computation and Machine Learning Series. MIT Press, Cambridge, MA, 1998.

6. M. Steingrover, R. Schouten, S. Peelen, B. Bakker and E Nijhuis, "Reinforcement Learning of Traffic Ligh Controllers Adapting to Traffic Congestion." Proceedings of the Belgium-Netherlands Artificial Intelligence Conference , 2005, pp.216-223

7. M. Zhang, S. Zhao, J. Lv and Y. Qian, "Multi-phase Urban Traffic Signal Real-time Control with Multiobjective Discrete Differential Evolution," 2009 International Conference on Electronic Computer Technology, Macau, 2009, pp. 296-300.

8. F. Wang, "Parallel Control and Management for Intelligent Transportation Systems: Concepts, Architectures, and Applications," in IEEE Transactions on Intelligent Transportation Systems, vol. 11, no. 3, pp. 630-638, Sept. 2010. doi: 10.1109/TITS.2010.2060218.

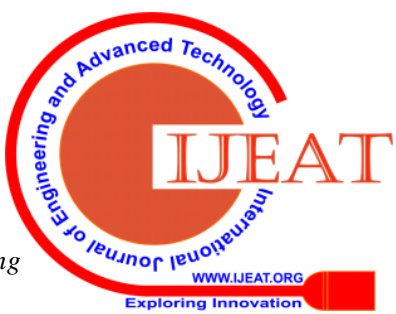


9. M. Turky, M. S. Ahmad, M. Z. M. Yusoff and N. R. Sabar, "Genetic Algorithm Application for Traffic Light Control," International United Information Systems Conference, Springer Berlin Heidelberg, 2009, pp. 115120.

10. S. Chen, C. Yang and Y. Peng, "Algorithms for the traffic light setting problem on the graph model," 2006. 